Trauma Berufskrankh 2008 · 10[Suppl 1]:3-8 DOI 10.1007/s10039-007-1219-2

Online publiziert: 14. April 2007

๑) Springer Medizin Verlag 2007

\section{W. Otto}

Universitätsklinik und Poliklinik für Unfall- und Wiederherstellungschirurgie,

Medizinische Fakultät, Martin-Luther-Universität, Halle, Saale

\title{
Historischer Abriss der Behandlung der verletzten Schulter
}

\section{Schulteranatomie und -funktion}

Die Schulter ist ein anatomisch-funktioneller Komplex aus stützenden, bewegungsaktiven, durchleitend ver- und entsorgenden sowie informationsvermittelnden Strukturen. Sie setzt sich zusammen aus:

- Schultergürtel,

- Klavikula mit angrenzenden Gelenken und

- Skapula.

Hinzu kommt das eigentliche Schultergelenk, gebildet aus:

- Glenoid und

- proximalem Humerus.

So konfiguriert, stellt die Schulter eine variable und zugleich stabile Basis für das Multifunktionsorgan „Arm und Hand“ dar, das als Sinnesorgan und Universalwerkzeug für uns Menschen eine immense Bedeutung hat. Die Schulter vermittelt dem Arm einen besonders großen dreidimensionalen Bewegungsumfang. Sie kann darüber hinaus Lastenträger sein und unterliegt wegen ihres multifunktionellen Gebrauchs einem hohen Verschleiß. Die starke Exposition führt zwangsläufig zu einer hohen Verletzungsanfälligkeit. In der Traumatologie wird die Schulter heute nach morphologisch-funktionellen Gesichtspunkten gegliedert betrachtet. Das gilt auch für die historische Entwicklung spezieller diagnostischer und therapeutischer Verfahren.

\section{Sternoklavikulargelenk}

Es stellt die einzige stützend-stabile Verbindung zwischen Rumpf, Schultergürtel und Arm dar. Es ist der Fixpunkt für die so genannte Mittelmastfunktion des Schlüsselbeins und seiner für die Armfunktion notwendigen Bewegungen. Es enthält als elastischen Puffer einen Discus articularis.

Das Sternoklavikulargelenk kann unter großer Beanspruchung luxieren, in der Regel nach vorn, weniger häufig nach oben und relativ selten nach hinten. Im letzteren Fall drohen vaskuläre oder mediastinale Begleitverletzungen.

Die Therapie der Luxation ist in der Regel konservativ-funktionell. Operative Maßnahmen sind bei hinterer Luxation zu empfehlen, bei Komplikationen zwingend. In der Literatur finden sich diverse Empfehlungen zu Fesselungsoperationen unter Verwendung von autogener Fascia lata, Drähten oder neuerdings so genannten Gelenkplatten. Bei Verrenkungsbrüchen sollten die Osteosynthesen das Gelenk nicht übergreifen. Es würden sonst eine Behinderung der Beweglichkeit des Schultergürtels und damit auch des Arms resultieren.

\section{Schlüsselbein}

\section{Grundlagen}

Die Klavikula weist eine Reihe von Besonderheiten auf, die bei der Gesamtbetrachtung ihrer Verletzungen zu berücksichtigen sind. Es handelt sich um einen des- mogenen Knochen. Epiphysenfugen, Epiund Metaphyse sowie ein röhrenförmiger Schaft mit einer echten Markhöhle fehlen hier also. Das Schlüsselbein unterliegt einer multidirektionalen Beanspruchung auf Zug, Stauchung, Rotation und Biegung und hat eine durchgehend suffiziente Durchblutung. Andererseits ist es in ganzer Länge nach vorn und oben lediglich von Haut bedeckt und hat nach dorsokaudal eine unmittelbare Nachbarschaft zu verletzlichen Strukturen, dem nervalen und dem vaskulären Plexus axillaris. Unter relativer Ruhigstellung heilt es in der Regel schnell und spontan unter Bildung eines kugelförmigen Kallus aus.

\section{Therapie von Verletzungen}

Beim Blick in die Geschichte fällt auf, dass die Behandlungsempfehlungen immer wieder zwischen konservativen und operativen Maßnahmen der verschiedensten Art schwankten. Eine wirkliche Beschleunigung der Heilung oder eine verbesserte therapeutische Sicherheit wurden dadurch jedoch nicht erreicht. Man findet eine Vielfalt von Verbandsanordnungen, meist unter Einbeziehung des Arms. Wie wir heute wissen, führt das aber eher zur Entwicklung von die Heilung störenden Bewegungen und Kräften. Auch aufwändige Schienen wurden zeitweilig empfohlen.

Die beste konservative Behandlungsmethode ist für uns bis heute der einfache, allerdings gut sitzende, anfangs deshalb engmaschig zu kontrollierende und zu korrigierende Rucksackverband. Bei Lorenz Böhler finden sich vergleichbare 
Empfehlungen, aber auch solche zur operativen Behandlung mittels Drähten oder Nägeln. Der Rucksackverband hat sich bis heute bewährt. Er bewirkt eine milde Extension bei relativer Ruhigstellung und führt so zur Kallusheilung.

In der operativen Behandlung verwendet man heute nicht mehr Drahtnähte, isolierte Kirschner-Drähte oder andere Implantate zur reinen inneren Schienung. Die Rekonstruktionsplattenosteosynthese, kranial oder ventral, kann inzwischen als Standardmethode angesehen werden. Eine reine innere Schienung dagegen erscheint uns nicht adäquat. Wir glauben, dass hierzu verriegelbare Implantate verwendet werden sollten.

Frakturen der peripheren Klavikula wurden früher ebenfalls überwiegend konservativ immobilisierend über 3-6 Wochen behandelt. Operative Maßnahmen mit Drähten oder Schlingen kamen wahlweise zur Anwendung. Heute klassifizieren wir die Verletzung nach Jäger und Breitner. Die Behandlung erfolgt bei dislozierten Frakturen operativ unter Verwendung von „Zuggurtungen“, kleinen Formplättchen oder im Kindesalter durch Naht des kräftigen Periosts und kurzzeitige Ruhigstellung. Nichtdislozierte Frakturen können kurzzeitig ruhig gestellt und sehr bald funktionell weiter behandelt werden. werden.

\section{Resümee}

Heutzutage bedürfen Schlüsselbeinfrakturen einer differenzierten Behandlung. Mediales und laterales Segment werden vorwiegend operativ nach den Prinzipien für gelenknahe Frakturen, unkomplizierte Schaftfrakturen dagegen größtenteils konservativ behandelt. Wenn Schaftfrakturen operiert werden, bedarf es unserer Meinung nach einer wirklich stabilen Osteosynthese.

\section{Akromioklavikular- oder Schultereckgelenk (ACG)}

Es handelt sich um ein echtes Gelenk mit einem Discus articularis und einem Kapsel-Band-Apparat sowie zusätzlichen kräftigen Bändern zwischen dem Processus coracoideus und der Klavikula.

Die Verletzung dieses Gelenks kann man in Distorsionen (Zerrung oder parti- elle Zerreißung von Kapsel und Bändern) entsprechend den Verletzungsstufen I und II nach Tossy und Rockwood unterteilen. Bei einer kompletten Luxation des Schultergelenks sind die Bandverbindungen und die Gelenkkapsel regelrecht zerrissen, entsprechend einer Verletzung vom Typ Tossy III oder Rockwood III-VI.

\section{Therapie von Verletzungen}

Man kann die therapeutischen Möglichkeiten zur Behandlung von ACG-Verletzungen bzw. -sprengungen in 4 Gruppen zusammenfassen: die konservativ-immobilisierenden, die konservativ-funktionellen, die operativ-immobilisierenden und die operativ-funktionellen Maßnahmen oder Konzepte.

\section{Konservative Behandlung}

Konservativ-immobilisierend. Auf diese Art versuchte man in der Vergangenheit durch Ruhigstellen auf Schienen (Apparat nach Bardenheuer u. Ä.) oder in Hartverbänden zu therapieren. Auch heute noch möglich und wirksam wären ein modifizierter Thoraxabduktionsgips für den Arm der verletzten Seite und ein Pflasterzügel, der die Klavikula nach unten hält. 4-6 Wochen lang getragen führt dies zu einer stabilen Ausheilung einer ACG-Ruptur.

Konservativ-funktionell. Da hinreichend bekannt ist, dass Menschen mit unbehandelter kompletter ACG-Sprengung keine bis wenig Beschwerden haben, kann man den Patienten eine konservativ-funktionelle Behandlung empfehlen. Sie besteht in einer kurzzeitigen Ruhigstellung in einem Armtragetuch oder Gilchrist-Verband und Freigabe der Funktion nach Abklingen des Verletzungsschmerzes. Es verbleibt zwar eine Instabilität mit dem typischen Klaviertastenphänomen, die Patienten sind davon jedoch nicht wesentlich beeinträchtigt. Lediglich bei Schwer- und Überkopfarbeitern sind die Ergebnisse nach operativer Behandlung wohl doch etwas besser als bei konservativer Therapie.

\section{Operative Behandlung}

Operative Methoden sind im vergangenen Jahrhundert in einer Vielzahl entworfen und entwickelt worden. Es gibt über 60 verschiedene operative Verfahren. In diesem Sinn besonders herauszustellen sind das Verfahren nach Bunnell, bei dem ein kompletter Ersatz des zerrissenen KapselBand-Apparats und der klavikulokorakoidalen Bänder durch einen Fascia-lataStreifen erfolgt, und jenes nach Bosworth, bei dem durch eine Stellschraube die Klavikula gegenüber dem Korakoid in Normalposition fixiert wird und dann der zerrissene Kapsel-Band-Apparat durch Naht adaptiert werden kann. Beide Verfahren erfordern eine zusätzliche äußere Ruhigstellung für bis zu 6 Wochen, da die erreichte Stabilität für die sofortige Freigabe der Funktion nicht ausreicht. Erst in den letzten Jahren steht nach Einführung der Hakenplatte eine gute und relativ sichere Methode zur Verfügung, die Klavikula gegenüber der Skapula unter Erhaltung der Beweglichkeit zu reponieren und dadurch die Heilung der zerrissenen Kapsel-BandStrukturen zu fördern. Von Kapsel-BandNähten und Augmentation durch PDSKordel ist eher abzuraten, da dabei nicht selten schwere Weichteilverknöcherungen eingetreten und beschrieben worden sind, die zur Ankylose des Schultereckgelenks führten.

\section{Resümee}

Insgesamt kann man die Therapieansätze auch heute noch nach ihrer Wertigkeit charakterisieren und nach Rücksprache mit dem Patienten gezielt einsetzen.

- Konservativ-immobilisierende Behandlung wird heute nur noch sehr selten gewünscht und akzeptiert. Absolute oder gute Indikationen dafür gibt es ebenfalls kaum.

- Konservativ-funktionell kann und sollte die Behandlung des „Normalbürgers" sein. Der Hinweis auf die verbleibende kosmetische Einschränkung allerdings gehört zum Aufklärungsgespräch.

- Die operativ-immobilisierenden Methoden sind heute ebenfalls wegen der notwendigen mehrwöchigen postoperativen Ruhigstellung weitgehend verlassen.

- Bevorzugt werden derzeit, wenn operativ behandelt werden soll, Methoden, die eine frühe Freigabe der Funktion des Arms und der Schulter erlauben. 


\section{„Forequarter-Amputation"}

Sie ist eine Sonderform der Schulterverletzung, häufig mit kompletter Zerreißung des ACG. Sie kommt offen oder geschlossen vor und besteht in einer kompletten Ruptur des Schultereckgelenks oder Frakturen der peripheren Klavikula sowie einer Nerven- und Gefäßverletzung unterschiedlichen Schweregrads. Sie stellt eine unmittelbare Bedrohung für die Erhaltung der Extremität dar und geht mit einer meist irreversiblen, teilweisen oder vollständigen Plexuslähmung einher. Erst moderne Diagnose- und Therapieverfahren haben zumindest Teilerfolge bei der Behandlung solcher Verletzungen möglich gemacht. Die Zusammenarbeit von Unfall, Gefäß-, plastischen und/oder Neurochirurgen ist für die Behandlung einer solchen Läsionen dringend zu fordern.

\section{Skapulafrakturen}

Die Skapula, das Schulterblatt, ist im Bereich der Squama von kräftigen Muskeln eingehüllt, die sowohl auf der dorsalen Oberfläche als auch auf der ventralen bzw. thorakalen Seite liegen.

Frakturen der Schulterblattschuppe sind deshalb in aller Regel konservativ zu behandeln. Auch Fortsatzfrakturen konnten bis zur Mitte des vorigen Jahrhunderts nur konservativ durch Ruhigstellung zur Heilung gebracht werden. Hierfür waren Ruhigstellungszeiten bis zu 6 Wochen erforderlich. Bis zur Mitte des vergangenen Jahrhunderts kamen operative Maßnahmen eigentlich nur in Form von Arthroplastiken oder Arthrodesen zum Einsatz, eher spät sekundär wegen Fehlheilung und konsekutiver Arthrose.

Heute werden auch die Frakturen das Schulterblatts differenzierter behandelt. Noch immer gibt es die Möglichkeit, konservativ-funktionell die Heilung problemlos herbeizuführen, sofern es sich um stabile nicht wesentlich dislozierte Frakturen handelt. Operativ sind im Allgemeinen dislozierte Fortsatzfrakturen und in seltenen Fällen verhakte oder offene Schulterblattschuppenbrüche zu behandeln. Von Bedeutung ist der so genannte superiore Suspensorkomplex der Schulter. Es handelt sich hierbei um die Ringkonstruktion, die sich aus Akromion, Pro-

Trauma Berufskrankh 2008 · 10[Suppl 1]:3-8 DOI 10.1007/s10039-007-1219-2

c) Springer Medizin Verlag 2007

W. Otto

Historischer Abriss der Behandlung der verletzten Schulter

\section{Zusammenfassung}

Bis etwa zur Mitte bzw. der zweiten Drittelgrenze des vergangenen Jahrhunderts standen zur Behandlung von Verletzungen der Schulter überwiegend konservative Maßnahmen zur Verfügung. Die Ergebnisse nach äuBerer Ruhigstellung in Schienen, fixierenden Apparaten oder Hartverbänden über 3-6 Wochen waren häufig nicht befriedigend. Erst mit der Einführung wirklich suffizienter Operationsverfahren konnten die verschiedenen Verletzungen gezielt einer Rekonstruktion und funktionellen Wiedererstellung zugeführt werden mit wesentlich besseren funktionellen und anatomischen Ergebnissen. Auch Wünsche der Patienten sowie ökonomisch begründete Vorgaben der Krankenh- ausgeschäftsleitungen und Versicherungen sind zu berücksichtigen. Die konservativen Verfahren werden immer mehr in den Hintergrund gedrängt und sind zu einem großen Teil bereits vollständig in Vergessenheit geraten. Sich ihrer zu erinnern, bedeutet jedoch auch, die durch operative Verfahren erreichbar gewordenen Erfolge einer konkreten Würdigung zu unterziehen.

\section{Schlüsselwörter}

Anatomisch-funktioneller Komplex . Historische Entwicklung · Spezifische Therapieansätze $\cdot$ Konservative

Möglichkeiten · Aktuelle Standards

\section{A brief historical account of the treatment of shoulder injuries}

\section{Abstract}

Up to halfway or two thirds of the way through the last century, the treatments available for the treatment of shoulder injuries were mainly conservative, and in many cases the results observed after external immobilization in different splints, fixation devices or plaster casts for 3 or more weeks were not satisfactory. With the development and introduction of really adequate operative methods it became possible to perform reconstruction and functional rehabilitation tailored to the different injuries, with much better anatomical and functional results. Due consideration must also be given to the patients' wishes and any economic restrictions imposed by hospital managements and healthcare insurances. Conservative measures are being pushed more and more into the background, and a good many of them are already completely forgotten. Remembering them also evokes appropriate appreciation of the successes that have become possible through operative procedures.

\section{Keywords}

Anatomic functional complex · Historical development $\cdot$ Specific therapeutic approaches . Conservative options - Current standards 
cessus coracoideus und dem oberen Pol des Glenoids bzw. dem oberen Rand des Schulterblatts an der Basis des Gelenkfortsatzes ergibt. Man sieht an dieser Linie v. a: die intakte Beschaffenheit des ACGelenks und evtl. Frakturen von Korakoid und Akromion. Die Osteosynthesen der Skapulafortsätze werden im Allgemeinen mit Schrauben- bzw. Schrauben und Platten durchgeführt.

Die so genannte "floating shoulder“, das gemeinsame Auftreten einer Klavikulafraktur und einer Fraktur des Schulterblatthalses oder des Glenoids, kann u. U. durch alleinige stabile Osteosynthese der Klavikula ausreichend reponiert und stabilisiert werden. Hier ist also nicht immer ein operatives Vorgehen an beiden Frakturen erforderlich.

\section{Rotatorenmanschette}

Die Mm. supraspinatus, infraspinatus und subscapularis sowie der vom lateralen Schulterblattrand her kommenden M. teres minor bilden miteinander die so genannte Rotatorenmanschette. Deren Aufgabe sind die Zentrierung und Fixation des Caput humeri gegenüber dem Glenoid als Voraussetzung für eine normale Funktion der äußeren thorakohumeralen Muskelmanschette. Um die Mitte des vergangenen Jahrhunderts und bis in dessen zweite Hälfte hinein waren die Erkrankungen der Rotatorenmanschette noch unter dem Begriff der „Periarthritis humeroscapularis“ zusammengefasst. Heute wissen wir diesen Komplex exakter einzuschätzen und sprechen von einer Rotatorenmanschettenläsion unterschiedlichen Grades.

\section{Diagnose}

Durch Arthroskopie, Sonographie, Radiometrie und das MRT sind exakte Befunderhebung und differenzierte Analyse chronisch sich entwickelnder und akut ggf. kurz vorher aufgetretener Beschwerdekomplexe und Funktionsstörungen möglich. Seitdem ist auch sicher, dass Schäden an der Rotatorenmanschette ganz überwiegend auf degenerative Veränderungen zurückgehen und in aller Regel erst durch das Vorhandensein solcher Vorschäden ein zusätzlicher Schaden durch äußere Einwirkung entstehen kann. In den allermeisten Fällen ist überwiegende Ursache der degenerative Schaden, eine überwiegend traumatische Ursache der Rotatorenmanschettenverletzungen oder -läsionen ist dagegen nur sehr selten nachweisbar und anzuerkennen.

\section{Therapie}

Die „Periarthritis humeroscapularis“ der 196oer und 1970er Jahre des vergangenen Jahrhunderts wurde konservativ-antiphlogistisch-analgetisch und ggf. durch $\mathrm{Ru}$ higstellungsmaßnahmen behandelt. Die Erfolge waren unbefriedigend, weshalb sich dann in den 1970er und 1980er Jahren die offene operative Therapie mehr und mehr entwickelte und zur Rekonstruktion oder auch im Sinne von Palliativmaßnahmen eingesetzt wurde. Auch diese Erfolge waren nicht überzeugend, weil auch hier postoperative Ruhigstellungen nötig waren und damit Verklebungen des Gelenks zu Funktionseinschränkungen nach der Behandlung führten. Heute wird die Rotatorenmanschettenläsion ganz überwiegend arthroskopisch therapiert. Dies geschieht minimalinvasiv und mit hoher Effizienz.

\section{Outcome}

Die funktionellen Ergebnisse sind auch heute noch nicht überzeugend. Das Problem liegt darin begründet, dass der $\mathrm{Pa}$ tient vor dem zusätzlichen Schadensereignis von seinem schon vorbestehenden Rotatorenmanschettendefekt nichts wusste und auch keine Beschwerden gehabt haben muss. Durch die nun unfallbedingt veränderte Situation wird die Symptomatik erst aufgedeckt. Kausalgenetisch ist jedoch der vorbestehende degenerative Schaden überwiegend heranzuziehen.

\section{Schultergelenk}

Es wird zwischen Processus glenoidalis mit seiner relativ kleinen Gelenkfläche und dem sehr viel größeren Caput humeri gebildet. Es ist als ein Kugelgelenk angelegt mit einer ausgeprägten Inkongruenz in Größe und Form der Gelenkpartner. Es wird überwiegend muskulär stabilisiert, geführt und zentriert und verbin- det großen Bewegungsumfang mit adäquater Stabilität. Dazu wird ein nachgiebiger, also nicht zu straffer Kapsel-BandApparat benötigt.

\section{Verletzungen}

Kapsel-Band-Schäden wie bei anderen Gelenken im Sinne einer Distorsion sind am Schultergelenk eher selten. Häufig vorkommende Verletzungen dagegen sind wegen der beschriebenen anatomisch-funktionellen Diskrepanz die Luxationen. Sie führen in aller Regel auch zu Schäden am Gelenk selbst in Form von Kapselschäden, Labrumablösungen oder anderen Avulsionen und Rupturen. Als zweite Verletzungsform kommen Luxationsfrakturen in Betracht. Diese können sowohl das Glenoid als auch den $\mathrm{Hu}$ meruskopf betreffen. Auf die Möglichkeit der begleitenden Gefäß- und Nervenverletzungen sei hier besonders hingewiesen. Sie ergibt sich aus der unmittelbaren Nähe der Armplexus zum Humeruskopf. Mit Hilfe von CT, MRT und Arthroskopie kann man heute derartige begleitende strukturelle Schäden sehr gut darstellen und identifizieren. Die Arthroskopie erlaubt uns heute auch, strukturelle Schäden frühzeitig zu erkennen und minimalinvasiv zu rekonstruieren.

Wir unterscheiden 3 vordere und eine hintere Luxationsmöglichkeit. Als Sonderform der axillaren Luxation ist die seltene Luxatio erecta zu erwähnen.

\section{Therapie}

Unter den Repositionsmanövern für Schulterverrenkungen ist heute immer noch die von Hippokrates empfohlene Methode in Gebrauch. Des Weiteren sind möglich die Verfahren nach Kocher, Arlt, Mothe u. a. Schulterluxationen waren offensichtlich schon immer ein Problem der Menschheit. Es gibt aus den verschiedensten Epochen der modernen Zeitrechnung Abbildungen, die die Verwendung z. B. der Methode nach Hippokrates darstellen. Nach der Reposition der Schulterluxation folgte allgemein eine Ruhigstellung von 3 Wochen, nur in seltenen Ausnahmefällen länger. Bereits nach dieser Zeit kommt es allgemein zu einer deutlichen Einsteifung der Schultern, die nur durch lang- 
Hier steht eine Anzeige.

并 Springer 
wierige und intensive Übungsbehandlung wieder verbessert werden kann.

Früher wurde die Rekonstruktion von Begleitverletzungen nicht primär durchgeführt. Sie wurden in der Regel erst bei auftretenden Komplikationen oder Folgeschäden aufgedeckt. Häufig waren dann umfangreiche und schwierige stabilisierende Eingriffe notwendig, oft mit funktionellen Verlusten und in der Regel mit unsicherem Erfolg.

Heute differenzieren wir bei jeder Schultergelenkerstluxationen nach Alter, Leistungsanspruch, klinischem, arthroskopischem und radiologischem Befund, den damit festgestellten Begleitschäden und natürlich dem Wunsch des Patienten. Zusammengefasst lässt sich feststellen:

- Junge, aktive Patienten mit knöchernen Begleitverletzungen sollten vorzugsweise operativ behandelt werden. - Ältere Patienten mit kapsuloligamentären Schäden sollten überwiegend eine konservative Therapie erhalten.

Trennlinie zwischen Jung und Alt ist heute noch bei etwa 30-40 Jahren. Weicht man von diesem Therapieschema sehr weit ab, kann es zu Rezidiven kommen. Bei älteren Patienten können die Vorteile operativer Verfahren kaum noch genutzt werden. Hier ist in aller Regel eine konservative Vorgehensweise ausreichend.

Die Arthroskopie hat auch bei der Schulter in Diagnostik und Therapie einen revolutionären Umschwung eingeleitet. Die gezielte Wiederherstellung oder Frührekonstruktion struktureller Schäden ist durch sie in aller Regel minimalinvasiv möglich. Dadurch können Rezidive und chronische Instabilitäten vermieden werden. Dies gilt sowohl für die Kapsel-BandSchäden als auch für knöcherne Läsionen wie die Bankart-Läsionen und den HillSachs-Defekt.

Durch die verbesserten Ergebnisse der Primärbehandlung können Korrektureingriffe heute weitgehend vermieden werden, die der Wiederherstellung oder Korrektur der Anatomie und der Restitution von Stabilität und Funktion dienen.

Bankart-Operationen werden heute in aller Regel primär geschlossen durchgeführt, sekundär sind sie kaum noch erforderlich. Spananlagerungen, z. B. nach Eden-Hybinette, kommen kaum noch in
Betracht. Das Gleiche gilt für die Osteotomie des Processus coracoideus nach Rüedi-Trillat und die subkapitale Rotationsosteotomie des Humerus nach Weber. Auch Korrektureingriffe an Weichteilstrukturen sind an Häufigkeit deutlich zurückgegangen. Das gilt auch für den Eingriff nach Putti-Platt an der Subskapularissehne sowie Fesselungsoperationen des Caput humeri mit der langen Bizepssehne (nach Bristow-Latarjet und Nicola-Heymanowitsch) oder die Fesselung mit Faszienstreifen nach Löffler.

\section{Humeruskopffrakturen}

Sie wurden bis vor 50 Jahren fast ausschließlich konservativ durch Ruhigstellung im Gipsverband behandelt. Danach aufgetretenen Schäden wurden durch Arthroplastik oder Prothese zu bessern gesucht.

Die Entwicklung der Plattenosteosynthese und bestimmter Formplatten (z. B. T-Platte der AO) brachte eine Wende zu ersten operativen Versorgungsstandards. Wegen ungünstiger Nebeneffekte der Plattenosteosynthese folgten Empfehlungen zur minimalinvasiv-operativen Therapie mittels Schrauben und Drähten. Diese beinhaltet jedoch ein Problem in der erreichten Stabilität. Heute sind Humeruskopffrakturen zunehmend eine Domäne der winkelstabilen Plattenosteosynthese. Auch Verriegelungsnägel für den proximalen Humerus kommen mehr und mehr zum Einsatz. Bei Mehrfragmentund Trümmerbrüchen, besonders beim Vorliegen erkennbar avaskulärer Fragmente, ist bereits primär an den partiellen Gelenkersatz zu denken, ganz speziell bei betagten Patienten, denen frühzeitig eine ausreichende, möglichst schmerzfreie Funktion wiedergegeben werden sollte.

\section{Schlussfolgerungen}

Wenn die Schulter - ein Funktionskomplex, der wichtig verletzt und schmerzt, auch funktioniert nicht richtig, erfordert dieses wohldurchdachte Prozeduren bei so viel unterschiedlichen Strukturen! Konservativ, sofern die Aussicht aufs Ergebnis gut, operativ seit neuerem, was nicht zuletzt darauf beruht, dass schlechte Resultate heute nicht mehr toleriert und manches eben besser heilt, rechtzeitig richtig operiert.

Historie ist Erfahrung und begründet Tradition,

auf die gestützt in gut geführten Häu-

sern immer schon

der Fortschritt in der Therapie gesucht mit Gründlichkeit, abgestimmt tradiertes Wissen mit Erkenntnissen der Zeit.

Moderne Therapie bedeutet eben, auch der verletzten Schulter eine echte Heilungschance zu geben, fortzuentwickeln die Verfahren und Methoden Schritt für Schritt, wie jeder ältere Chirurg es hat erlebt und auch gestaltet mit.

\section{Korrespondierender Autor}

Prof. Dr. W. Otto

Universitätsklinik und Poliklinik für Unfall- und Wiederherstellungschirurgie, Medizinische

Fakultät, Martin-Luther-Universität,

Ernst-Grube-Straße 40, 06097 Halle, Saale

wieland.otto@medizin.uni-halle.de

Interessenkonflikt. Es besteht kein Interessenkonflikt. Der korrespondierende Autor versichert, dass keine Verbindungen mit einer Firma, deren Produkt in dem Artikel genannt ist, oder einer Firma, die ein Konkurrenzprodukt vertreibt, bestehen. Die Präsentation des Themas ist unabhängig und die Darstellung der Inhalte produktneutral.

Literatur

... beim Verfasser 